\title{
EINE ZUR PARALLELOGRAMMGLEICHUNG ÄQUIVALENTE UNGLEICHUNG
}

\author{
ATTILA GILÁNYI
}

\begin{abstract}
Meinem sehr verehrten Lehrer und Doktorvater, Herrn Professor Dr. Peter Volkmann, anläßlich seines sechzigsten Geburtstages gewidmet
\end{abstract}

Zusammenfassung. In der vorliegenden Arbeit wird bewiesen, daß für eine auf einer durch 2 teilbaren abelschen Gruppe $G$ definierte, in einen Skalarproduktraum $E$ abbildende Funktion $f$ aus der Funktionalungleichung

$$
\left\|2 f(x)+2 f(y)-f\left(x y^{-1}\right)\right\| \leq\|f(x y)\| \quad(x, y \in G)
$$

folgt, daß $f$ die sogenannte Parallelogrammgleichung

$$
f(x y)+f\left(x y^{-1}\right)-2 f(x)-2 f(y)=0 \quad(x, y \in G)
$$

erfüllt.

$$
\begin{aligned}
& \text { Abstract. In this paper it is proved that, for a function } f: G \rightarrow E \text { map- } \\
& \text { ping from an abelian group divisible by } 2 \text { into an inner product space } E \text {, the } \\
& \text { inequality } \\
& \qquad\left\|2 f(x)+2 f(y)-f\left(x y^{-1}\right)\right\| \leq\|f(x y)\| \quad(x, y \in G) \\
& \text { implies } \\
& \qquad f(x y)+f\left(x y^{-1}\right)-2 f(x)-2 f(y)=0 \quad(x, y \in G) .
\end{aligned}
$$

P. Volkmann hat in seinem Vortrag [11] auf der dreiundzwanzigsten Internationalen Tagung über Funktionalgleichungen (Gargnano, Italien, 1985) gezeigt, daß für eine reelle Funktion $f$ aus der Ungleichung

$$
|f(x)+f(y)| \leq|f(x+y)| \quad(x, y \in \mathbb{R})
$$

Schlüsselwörter: Funktionalgleichungen, Funktionalungleichungen, Parallelogrammgleichung. $M S C$ (2000): 39B62. 
folgt, daß $f$ die Cauchysche Funktionalgleichung

$$
f(x+y)=f(x)+f(y) \quad(x, y \in \mathbb{R})
$$

erfüllt. Dieses Ergebnis wurde von S. Kurepa [4] folgendermaßen verallgemeinert. Ist $G$ eine Gruppe, $E$ ein Skalarproduktraum und $f: G \rightarrow E$ eine Funktion, die die Modifikation

$$
\|f(x y z)\|=\|f(x z y)\| \quad(x, y, z \in G)
$$

der sogenannten Kannappan-Bedingung (vgl. [3])

$$
f(x y z)=f(x z y) \quad(x, y, z \in G)
$$

und die Ungleichung

$$
\|f(x)+f(y)\| \leq\|f(x y)\| \quad(x, y \in G)
$$

erfüllt, dann gilt

$$
f(x y)=f(x)+f(y) \quad(x, y \in G) .
$$

Gy. Maksa und P. Volkmann [7] haben diesen Satz ohne die Annahme (1) bewiesen (vgl. auch [6]). Motiviert durch diese Ergebnisse untersuchen wir zu (2) ähnliche „Norm-Ungleichungen“, die sich aus der Parallelogrammgleichung (vgl. u.a. [1], [2], [5], [10]) bilden lassen.

In ihrer Arbeit [8] hat F. Skof das folgende Resultat gezeigt. Falls $X$ ein reeller linearer Raum ist und für eine Funktion $f: X \rightarrow \mathbb{R}$ eine der Gleichungen

$$
\begin{array}{ll}
|f(x+y)|=|2 f(x)+2 f(y)-f(x-y)| & (x, y \in X) \\
|f(x-y)|=|2 f(x)+2 f(y)-f(x+y)| & (x, y \in X) \\
|f(x+y)+f(x-y)-2 f(x)|=|2 f(y)| & (x, y \in X) \\
|f(x+y)+f(x-y)-2 f(y)|=|2 f(x)| & (x, y \in X) \\
|f(x+y)+f(x-y)|=|2 f(x)+2 f(y)| & (x, y \in X)
\end{array}
$$

gilt, so erfüllt $f$ die Parallelogrammgleichung

$$
f(x+y)+f(x-y)-2 f(x)-2 f(y)=0 \quad(x, y \in X) .
$$

In [9] hat F. Skof bewiesen, daß für eine Funktion $f$, die in einen Skalarproduktraum $E$ abbildet, die Gleichungen (3), (4) und (8) zueinander äquivalent sind (wo $|$.$| die$ Norm in $E$ bezeichnet).

In der vorliegenden Arbeit beweisen wir unter Verwendung ähnlicher Methoden wie in [8] und [9], daß für eine auf einer durch 2 teilbaren abelschen Gruppe $G$ definierte und in einen Skalarproduktraum $E$ abbildende Funktion $f$ aus

$$
\left\|2 f(x)+2 f(y)-f\left(x y^{-1}\right)\right\| \leq\|f(x y)\| \quad(x, y \in G)
$$

folgt, daß $f$ die Parallelogrammgleichung

$$
f(x y)+f\left(x y^{-1}\right)-2 f(x)-2 f(y)=0 \quad(x, y \in G)
$$


erfüllt. (Wir deuten auch darauf hin, daß in unserem Beweis die Kommutativität von $G$ durch die Kannappan-Bedingung (1) ersetzt werden kann. Das ist der Grund dafür, daß wir in $G$ die multiplikative Schreibweise benutzen; vgl. Bemerkung 1.) Durch Angabe von Gegenbeispielen zeigen wir, daß für die anderen NormUngleichungen, die sich aus der Parallelogrammgleichung bilden lassen, keine solche Behauptung gilt.

Satz 1. Es sei $G$ eine durch 2 teilbare abelsche Gruppe, E ein Skalarproduktraum. Gilt für eine Funktion $f: G \rightarrow E$

$$
\left\|2 f(x)+2 f(y)-f\left(x y^{-1}\right)\right\| \leq\|f(x y)\| \quad(x, y \in G),
$$

dann erfüllt sie die Funktionalgleichung

$$
f(x y)+f\left(x y^{-1}\right)-2 f(x)-2 f(y)=0 \quad(x, y \in G) .
$$

Beweis. Wir führen den Beweis für einen reellen Skalarproduktraum $E$ durch. Die Behauptung für einen komplexen Skalarproduktraum $E$ folgt daraus.

Ersetzt man $x$ und $y$ in (9) durch das Einheitselement $e$ von $G$, so bekommt man

also

$$
3\|f(e)\| \leq\|f(e)\|
$$

das heißt

$$
\|f(e)\| \leq 0
$$

$$
f(e)=0 .
$$

Die Substitution $y:=x^{-1}$ in (9) liefert

$$
\left\|2 f(x)+2 f\left(x^{-1}\right)-f(x x)\right\| \leq 0 \quad(x \in G),
$$

woraus

$$
2 f(x)+2 f\left(x^{-1}\right)=f(x x) \quad(x \in G)
$$

folgt. Mit $x^{-1}$ statt $x$ ergibt sich daraus

$$
2 f\left(x^{-1}\right)+2 f(x)=f\left(x^{-1} x^{-1}\right) \quad(x \in G) .
$$

Subtrahiert man diese Gleichung von (12), dann erhält man

$$
f(x x)=f\left(x^{-1} x^{-1}\right) \quad(x \in G),
$$

also, wegen der Teilbarkeit der Gruppe $G$ durch 2,

$$
f(x)=f\left(x^{-1}\right) \quad(x \in G) .
$$

Aus (12) und (13) folgt

$$
f(x x)=4 f(x) \quad(x \in G) .
$$

Durch Quadrieren der beiden Seiten von (9) ergibt sich

$$
\left\|2 f(x)+2 f(y)-f\left(x y^{-1}\right)\right\|^{2} \leq\|f(x y)\|^{2} \quad(x, y \in G),
$$


das heißt

$$
\begin{array}{r}
\|2 f(x)+2 f(y)\|^{2}+\left\|f\left(x y^{-1}\right)\right\|^{2}-2 \\
\left.\leq 2 f(x)+2 f(y), f\left(x y^{-1}\right)\right\rangle \\
\leq\|f(x y)\|^{2} \quad(x, y \in G),
\end{array}
$$

wobei $\langle.,$.$\rangle das Skalarprodukt in E$ bezeichnet. Ersetzt man in dieser Ungleichung $y$ durch $y^{-1}$, so bekommt man

$\left\|2 f(x)+2 f\left(y^{-1}\right)\right\|^{2}+\|f(x y)\|^{2}-2\left\langle 2 f(x)+2 f\left(y^{-1}\right), f(x y)\right\rangle \leq\left\|f\left(x y^{-1}\right)\right\|^{2}(x, y \in G)$.

Nach Anwendung von (13) und nach Addition der letzten beiden Ungleichungen ergibt sich

$$
2\|2 f(x)+2 f(y)\|^{2}-2\left\langle 2 f(x)+2 f(y), f(x y)+f\left(x y^{-1}\right)\right\rangle \leq 0 \quad(x, y \in G),
$$

oder umgeformt

$$
\begin{aligned}
& \langle-2 f(x)-2 f(y),-2 f(x)-2 f(y)\rangle \\
& +\left\langle-2 f(x)-2 f(y), f(x y)+f\left(x y^{-1}\right)\right\rangle \leq 0 \quad(x, y \in G),
\end{aligned}
$$

das heißt

$$
\left\langle-2 f(x)-2 f(y), f(x y)+f\left(x y^{-1}\right)-2 f(x)-2 f(y)\right\rangle \leq 0 \quad(x, y \in G) .
$$

Schreibt man anderseits $y^{-1} x^{-1}$ statt $x$ und $y x^{-1}$ statt $y$ in (15), so bekommt man

$$
\begin{aligned}
& \left\|2 f\left(y^{-1} x^{-1}\right)+2 f\left(y x^{-1}\right)\right\|^{2}+\left\|f\left(y^{-1} y^{-1}\right)\right\|^{2} \\
& -2\left\langle 2 f\left(y^{-1} x^{-1}\right)+2 f\left(y x^{-1}\right), f\left(y^{-1} y^{-1}\right)\right\rangle \leq\left\|f\left(y^{-1} x^{-1} y x^{-1}\right)\right\|^{2} \quad(x, y \in G),
\end{aligned}
$$

also, wegen (13) und (14),

$$
\begin{array}{r}
\left\|2 f(x y)+2 f\left(x y^{-1}\right)\right\|^{2}+\left\|f\left(y^{-1} y^{-1}\right)\right\|^{2}-2\left\langle 2 f(x y)+2 f\left(x y^{-1}\right), 4 f(y)\right\rangle \\
\leq\left\|f\left(y^{-1} x^{-1} y x^{-1}\right)\right\|^{2} \quad(x, y \in G) .
\end{array}
$$

Mit $y^{-1} x^{-1}$ statt $x$ und $x y^{-1}$ statt $y$ in (15) bekommen wir

$$
\begin{aligned}
& \left\|2 f\left(y^{-1} x^{-1}\right)+2 f\left(x y^{-1}\right)\right\|^{2}+\left\|f\left(y^{-1} x^{-1} y x^{-1}\right)\right\|^{2} \\
& -2\left\langle 2 f\left(y^{-1} x^{-1}\right)+2 f\left(x y^{-1}\right), f\left(y^{-1} x^{-1} y x^{-1}\right)\right\rangle \\
& \leq\left\|f\left(y^{-1} y^{-1}\right)\right\|^{2} \quad(x, y \in G) .
\end{aligned}
$$

Die Kommutativität von $G$, (13) und (14) liefern

$$
\begin{aligned}
\left\|2 f(x y)+2 f\left(x y^{-1}\right)\right\|^{2}+\left\|f\left(y^{-1} x^{-1} y x^{-1}\right)\right\|^{2}- & 2\left\langle 2 f(x y)+2 f\left(x y^{-1}\right), 4 f(x)\right\rangle \\
\leq & \left\|f\left(y^{-1} y^{-1}\right)\right\|^{2} \quad(x, y \in G) .
\end{aligned}
$$

Die Addition von (17) und (19) gibt

$$
8\left\|f(x y)+f\left(x y^{-1}\right)\right\|^{2}-8\left\langle f(x y)+f\left(x y^{-1}\right), 2 f(x)+2 f(y)\right\rangle \leq 0 \quad(x, y \in G),
$$

somit

$$
\begin{aligned}
& \left\langle f(x y)+f\left(x y^{-1}\right), f(x y)+f\left(x y^{-1}\right)\right\rangle \\
& +\left\langle f(x y)+f\left(x y^{-1}\right),-2 f(x)-2 f(y)\right\rangle \leq 0 \quad(x, y \in G),
\end{aligned}
$$


das ist

$$
\left\langle f(x y)+f\left(x y^{-1}\right), f(x y)+f\left(x y^{-1}\right)-2 f(x)-2 f(y)\right\rangle \leq 0 \quad(x, y \in G) .
$$

Die Addition dieser Ungleichung zu (16) liefert

$\left\langle f(x y)+f\left(x y^{-1}\right)-2 f(x)-2 f(y), f(x y)+f\left(x y^{-1}\right)-2 f(x)-2 f(y)\right\rangle \leq 0 \quad(x, y \in G)$,

das heißt

$$
\left\|f(x y)+f\left(x y^{-1}\right)-2 f(x)-2 f(y)\right\|^{2} \leq 0 \quad(x, y \in G),
$$

demzufolge gilt (10).

Bemerkung 1. Die Kommutativität der Gruppe $G$ wurde in dem obigen Beweis nur bei der Umformung der Ungleichung (18) benutzt. Es ist leicht zu sehen, daß es reicht, hier statt der Kommutativität die Kannappan-Bedingung (1) für $f$ anzunehmen. Dies führt zur offenen Frage, ob die Behauptung unseres Satzes ohne irgendeine Kommutativitäts-Bedingung gilt.

Bemerkung 2. Aus der Parallelogrammgleichung lassen sich dreizehn weitere, zu (9) ähnliche Ungleichungen bilden. Im folgenden zeigen wir, daß (bereits für reelle Funktionen) keine dieser Ungleichungen zur Parallelogrammgleichung äquivalent ist.

1. Betrachten wir zuerst die Ungleichung

$$
|2 f(x)+2 f(y)-f(x+y)| \leq|f(x-y)| \quad(x, y \in \mathbb{R}) .
$$

Es ist leicht nachzuweisen, daß mit einer reellen Konstante $c \neq 0$ die Funktionen $f: \mathbb{R} \rightarrow \mathbb{R}$,

$$
f(x)= \begin{cases}c x^{2} & \text { für } x \geq 0 \\ -c x^{2} & \text { für } x<0,\end{cases}
$$

die Ungleichung (20) erfüllen, die Parallelogrammgleichung aber nicht lösen. In der Tat, der zweite Teil der Behauptung ist trivial, der erste kann so gezeigt werden, daß man alle möglichen Fälle der Vorzeichen von $x, y, x+y$ und $x-y$ (insgesamt 8 ) untersucht. Zum Beispiel, falls $x>0, y<0$, und $x+y>0$ (somit auch $|x| \geq|y|)$ gilt, hat (20) die Form

$$
\left|2 x^{2}-2 y^{2}-(x+y)^{2}\right| \leq\left|(x-y)^{2}\right| \quad(x, y \in \mathbb{R}),
$$

das heißt

$$
\left|x^{2}+2 x\right| y\left|-3 y^{2}\right| \leq\left|x^{2}+2 x\right| y\left|+y^{2}\right| \quad(x, y \in \mathbb{R}) .
$$

Wegen $x^{2}+2 x|y|-3 y^{2} \geq x^{2}+2 y^{2}-3 y^{2} \geq 0$ kann das Betrag-Zeichen in den obigen Ungleichungen weggelassen werden, daher gelten diese Ungleichungen und (20) für die betrachteten $x$ und $y$.

2. Unter Verwendung des binomischen Satzes bekommt man, daß mit einer beliebigen ganzen Zahl $k>1$ und reellen Zahl $c \neq 0$ die Funktionen $f(x)=c x^{2 k}(x \in \mathbb{R})$ 
die Ungleichungen

$$
\begin{aligned}
|2 f(x)| & \leq|f(x+y)+f(x-y)-2 f(y)| \quad(x, y \in \mathbb{R}) \\
|2 f(y)| & \leq|f(x+y)+f(x-y)-2 f(x)| \quad(x, y \in \mathbb{R}) \\
|2 f(x)+2 f(y)| & \leq|f(x+y)+f(x-y)| \quad(x, y \in \mathbb{R})
\end{aligned}
$$

erfüllen. Für alle reellen Paare $(x, y)$ mit $x y \neq 0$ alle der obigen Ungleichungen mit ,$<$ " gelten.

3. Alle der übrigen 9 Funktionalungleichungen werden durch alle konstanten Funktionen erfüllt, die von 0 verschiedenen konstanten Funktionen lösen aber die Paralellogrammgleichung nicht.

Bemerkung 3. Wir deuten schließlich darauf hin, daß unser Satz für Halbgruppen auch dann nicht verallgemeinert werden kann, falls wir statt der Paralellogrammgleichung die auf Gruppen dazu äquivalente, sogenannte monomiale Funktionalgleichung zweiten Grades betrachten. Die Funktion $f:[0, \infty) \rightarrow \mathbb{R}, f(x)=c x^{k}$ erfüllt nämlich für eine belibige ganze Zahl $k>2$ und eine reelle Zahl $c \neq 0$ die Ungleichung

$$
|2 f(x+y)-f(x)+2 f(y)| \leq|f(x+2 y)| \quad(x, y \in[0, \infty)),
$$

sie ist aber keine Lösung von

$$
|2 f(x+y)-f(x)+2 f(y)|=|f(x+2 y)| \quad(x, y \in[0, \infty)) .
$$

\section{Danksagung}

Die Ergebnisse der vorliegenden Arbeit entstanden während meines Aufenthaltes an der Universität Karlsruhe. Für seine freundliche Gastfreundschaft und für die Anregung zu der Arbeit danke ich ganz herzlich Herrn Professor Dr. Peter Volkmann, für die finanzielle Unterstützung des Aufenthaltes gilt mein Dank dem Deutschen Akademischen Austauschdienst.

\section{LITERATURVERZEICHNIS}

[1] J. Aczél, The general solution of two functional equations by reduction to functions additive in two variables and with the aid of Hamel bases, Glasnik Mat.-Fiz. Astronom. Ser. II, 20(1965), 65-73.

[2] M. Hosszú, A remark on the square norm, Aequationes Math. 2(1969), 190-193.

[3] Pl. Kannappan, The functional equation $f(x y)+f\left(x y^{-1}\right)=2 f(x) f(y)$ for groups, Proc. Amer. Math. Soc. 19(1968), 69-74.

[4] S. Kurepa, On P. Volkmann's paper, Glas. Mat. Ser. III, 22(42)(1987), 371-374.

[5] Gy. Maksa, A remark on symmetric biadditive functions having nonnegative diagonalization, Glas. Mat. Ser. III, 15(35)(1980), 279-282.

[6] Gy. Maksa, Remark on the talk of P. Volkmann, Proc. of the Twenty-third International Symposium on Functional Equations (Gargano, Italy, 1985), Centre for Information Theory, Faculty of Mathematics, Oniversity of Waterloo, Waterloo, Ontario, Canada, 72-73.

[7] Gy. Maksa, P. Volkmann, Characterization of group homomorphisms having values in an inner product space, Publ. Math. Debrecen 56(2000), 197-200. 
[8] F. Skof, On some alternative quadratic equations, Results Math. 27(1995), 402-411.

[9] F. Skof, On some alternative quadratic equations in inner-product spaces, Atti Sem. Mat. Fis. Univ. Modena 46(1998), 951-962.

[10] H. Światak, On two functional equations connected with the equation $\varphi(x+y)+\varphi(x-y)=$ $2 \varphi(x)+2 \varphi(y)$, Aequationes Math. 5(1970), 3-9.

[11] P. Volkmann, Pour une fonction réelle $f$ l'inéquation $|f(x)+f(y)| \leq|f(x+y)|$ et l'équation de Cauchy sont équivalentes, Proc. of the Twenty-third International Symposium on Functional Equations (Gargnano, Italy, 1985), Centre for Information Theory, Faculty of Mathematics, University of Waterloo, Waterloo, Ontario, Canada, 43.

Institut für Mathematik und Informatik, Universität Debrecen, 4010 Debrecen, PF.:12, UNGARN

E-mail address: gil@math.klte.hu 